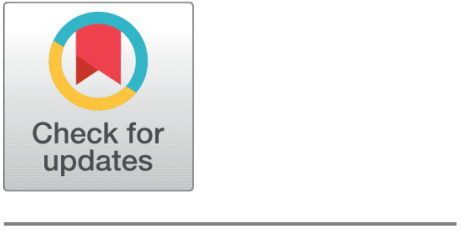

OPEN ACCESS

Received: 17.02.2021

Accepted: 06.04.2021

Published: 24.04.2021

Citation: Arputha Jose T, Daniel Raj A, Venugopal P, Giridaran M (2021) Radio antipodal mean number of quadrilateral Snake families. Indian Journal of Science and Technology 14(13): 1071-1080. https://doi.org/ 10.17485/IJST/V14i13.295

* Corresponding author.

Tel: +91-957885514

danielraj61@yahoo.com

Funding: None

Competing Interests: None

Copyright: (c) 2021 Arputha Jose et al. This is an open access article distributed under the terms of the Creative Commons Attribution License, which permits unrestricted use, distribution, and reproduction in any medium, provided the original author and source are credited.

Published By Indian Society for Education and Environment (iSee)

ISSN

Print: 0974-6846

Electronic: 0974-5645

\section{Radio antipodal mean number of quadrilateral Snake families}

\author{
T Arputha Jose ${ }^{1}$, A Daniel Raj ${ }^{1 *}$, P Venugopal ${ }^{2}$, M Giridaran ${ }^{3}$ \\ 1 Research Scholar, Department of Mathematics, Sri Sivasubramaniya Nadar College of \\ Engineering (Autonomous), Kalavakkam, 603110, Tamilnadu, India. Tel.: +91-957885514 \\ 2 Associate professor, Department of Mathematics, Sri Sivasubramaniya Nadar College of \\ Engineering (Autonomous), Kalavakkam, 603110, Tamilnadu, India \\ 3 Lecturer, Department of Mathematics, DMI-St. Eugene University, Lusaka, Zambia
}

\section{Abstract}

Objectives: In communication engineering, the assignment of channels or frequencies to different transmitters in a communication network without interference is an important problem. Finding the span for such an assignment is a challenging task. The objective of this study is to find the span of quadrilateral snake families. Method: The solution to the channel assignment problem can be found out by modeling the communication network as a graph, where the transmitters are represented by nodes and connectivity between transmitters are given by edges. The labeling technique in graph theory is very useful to solve this problem. Let $G=(V, E)$ be a graph with vertex set $\mathrm{V}$, edge set E. Let $u, v \in V(G)$. The radio antipodal mean labeling of a graph $\mathrm{G}$ is a function $\mathrm{f}$ that assigns to each vertex $u$, a non-negative integer $f(u)$ such that $f(u) \neq f(v)$ if $d(u, v)<\operatorname{diam}(G)$ and $d(u, v)+\left[\frac{f(u)+f(v)}{2}\right] \geq \operatorname{diam}(G)$, where $d(u, v)$ represents the shortest distance between any pair of vertices $u$ and $v$ of $G$ and $\operatorname{diam}(G)$ is the diameter of $\mathrm{G}$. The radio antipodal mean number of $\mathrm{f}$, is the maximum number assigned to any vertex of $\mathrm{G}$ and is denoted by $\operatorname{ramn}(f)$. The radio antipodal mean number of $\mathrm{G}$, denoted by $\operatorname{ramn}(G)$ is the minimum value of $\operatorname{ramn}(f)$ taken over all antipodal mean labeling $f$ of $G$. Findings: In this study, we have obtained the bounds of radio antipodal mean number of quadrilateral snake families. Novelty: The radio antipodal mean number of quadrilateral snake families was not studied so far. Hence, the establishment of the bounds for radio mean number of quadrilateral snake families will motivate many researchers to study the radio antipodal mean number of other communication networks.

Keywords: Radio antipodal mean labeling; quadrilateral snake; alternate quadrilateral snake; double quadrilateral snake; double alternate quadrilateral snake

\section{Introduction}

The radio labeling technique has a lot of application in communication engineering. It is mainly used to assign channels or frequencies to different radio stations. In each radio station, its antennas propagate electromagnetic waves with different frequencies, 
known as radio waves. A specific signal can be accessed, by tuning the radio receiver to a particular frequency. All the radio station must be assigned with distinct channels, located within a certain proximity of one another ${ }^{(1)}$. The level of interference is closely related to the geographical location of the stations - the closer are the stations, the stronger the interference between them. To avoid interference, the difference between the channels assigned to a pair of nearby stations must be large enough. The channels can be assigned to each radio station in a network, by modeling the network as a graph, where each station is represented by a vertex and the stations whose geographical locations are very close are joined by an edge ${ }^{(2)}$. The assignment of integers to the vertices, edges or both based on certain condition is known as graph labeling ${ }^{(3)}$.

The procedure of assigning channels (frequencies) efficiently to all radio transmitters is popularly known as the channel assignment problem ${ }^{(4)}$. William Hale formulated this problem as a graph coloring problem in $1980^{(5)}$. To solve the channel assignment problem, the interference graph is developed, and the assignment of channels has been converted into a graph coloring or graph labeling problem, where we assign different labels (positive integer) or colors to all the vertices in the graph such that the adjacent vertices receive different colors (or labels) ${ }^{(6)}$. Jerrold R. Griggs and Roger K. Yeh ${ }^{(7)}$ devised this problem as a distance 2 labeling or $L(2,1)$ labeling. It is defined as follows: Given a real number $d>0$, an $L_{d}(2,1)-$ labeling of $G$ is a non-negative real-valued function $f: V(G) \rightarrow(0, \infty)$ such that, whenever $x$ and $y$ are two adjacent vertices in $V$, then $|f(x)-f(y)| \geq 2 d$, and whenever the distance between $x$ and $y$ is 2 , then $|f(x)-f(y)| \geq d$.

The work of Jerrold et al. motivated Chartrand et al. ${ }^{(8)}$ to introduce a new graph labeling technique called radio labeling. The radio labeling of graphs is just an extension of $L(2,1)$ labeling. A radio labeling of a graph $G$ is a function $f: V(G) \rightarrow N$ such that, $d(u, v)+|f(u)-f(v)| \geq 1+\operatorname{diam}(G)$, where $d(u, v)$ represents the shortest distance between the vertices $u$ and $v$ and $\operatorname{diam}(G)$ is the diameter of $G$. The problem of finding the radio number of an arbitrary graph is proved to be an NPComplete problem ${ }^{(9)}$. In 2002, Chartrand et al. ${ }^{(10)}$ defined a new graph labeling technique called radio antipodal labeling by modifying the existing radio labeling definition. The radio antipodal labeling of a graph $G$ is a function $f: V(G) \rightarrow N$ such that $d(u, v)+|f(u)-f(v)| \geq \operatorname{dim}(G)$. The span obtained by radio antipodal labeling of a graph is less compared to radio labeling of a graph as the vertices at diametric distances are assigned the same label in antipodal labeling. In 2015, Ponraj et al. ${ }^{(11)}$ redefined the radio labeling condition and introduced a new graph labeling technique called radio mean labeling. A radio mean labeling of a graph $G$ is a one-to-one map $f$ from the vertex set $V(G)$ to the set of natural numbers $N$ such that for any two distinct vertices $u$ and $v$ of $G, d(u, v)+\left\lceil\frac{f(u)+f(v)}{2}\right\rceil \geq 1+\operatorname{diam}(G)$.

The radio antipodal labeling condition was modified by Xavier and Thivyarathi ${ }^{(12)}$ in 2018 and introduced a new graph labeling technique called radio antipodal mean labeling. The radio antipodal mean labeling of a graph $G$ is a function $f$ that assigns to each vertex $u$, a non-negative integer $f(u)$ such that $d(u, v)+\left\lceil\frac{f(u)+f(v)}{2}\right\rceil \geq \operatorname{diam}(G)$. If $d(u, v)<\operatorname{diam}(G)$, then $f(u) \neq f(v)$. In their work, they have investigated the radio antipodal mean number of paths, wheel, cycle, mesh, and its derived architectures. The radio antipodal mean number of certain types of ladder graphs have been studied by Yenoke et al. ${ }^{(13)}$ and Jose et al. ${ }^{(3)}$ obtained the upper bounds of radio antipodal mean number of triangular snake families. It was observed that the radio antipodal mean number is less than the radio antipodal number. The radio antipodal mean labeling technique reduces the span compared to radio antipodal labeling.

This paper has been further organized as follows. In section 2, the preliminaries necessary for our discussion were presented. The main results are discussed in section 3, followed by a Conclusion. In this paper, the bounds of radio antipodal mean number of quadrilateral snake families have been obtained.

\section{PRELIMINARIES}

In this section, the terminologies necessary for our study are presented.

Definition 2.1 ${ }^{(12)}$. The radio antipodal mean labeling of a graph $G$ is a function $f$ that assigns to each vertex $\mathrm{u}$, a non-negative integer $f(u)$ such that $f(u) \neq f(v)$ if $d(u, v)<\operatorname{diam}(G)$ and $d(u, v)+\left\lceil\frac{f(u)+f(v)}{2}\right\rceil \geq \operatorname{diam}(G)$, where $d(u, v)$ represents the shortest distance between any pair of vertices $u$ and $v$ of $G$.

Definition 2.2 ${ }^{(14)}$. A quadrilateral snake $Q S_{n}$ is obtained from a path $v_{1}, v_{2}, \ldots, v_{n}$ by joining $v_{i}$ and $v_{i+1}, 1 \leq i \leq n-1$, to new vertices $u_{j}$ and $u_{j+1}$ respectively and joining the vertices $u_{j}$ and $u_{j+1}$ for $j=1,3, \ldots, 2 n-3$. That is every edge of a path is replaced by a cycle $C_{4}$. For example, see Figure 1 . 


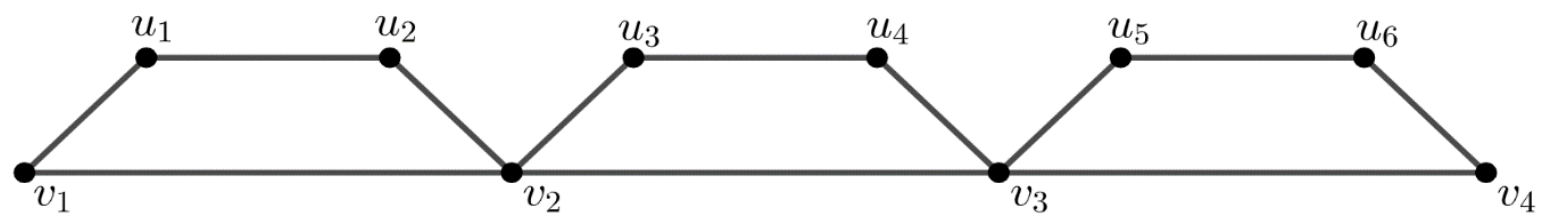

Fig 1. $Q S_{4}$

Definition $2.3^{(14)}$. An alternate quadrilateral snake $A Q S_{n}$ is obtained from a path $v_{1}, v_{2}, \ldots v_{n}$ by joining $v_{i}$ and $v_{i+1}$ to new vertices $u_{i}$ and $u_{i+1}$ respectively for $i \equiv 1(\bmod 2)$ and $i \leq n-1$ and then joining $u_{i}$ and $u_{i+1}$. That is every alternative edge of a path is replaced by a cycle $C_{4}$. For example, see Figure 2 .

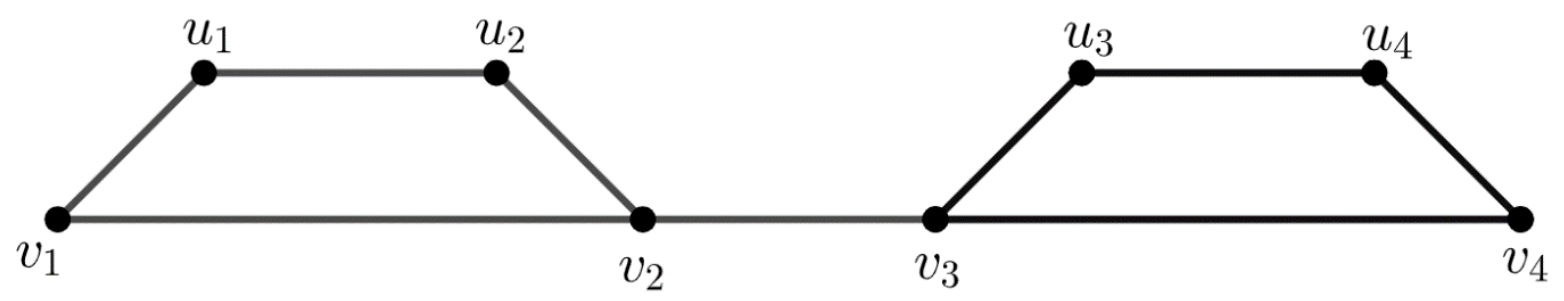

Fig 2. $A Q S_{4}$

Definition $2.4^{(14)}$. A double quadrilateral snake $D Q S_{n}$ is obtained from two quadrilateral snakes that have a common path. For example, see Figure 3.

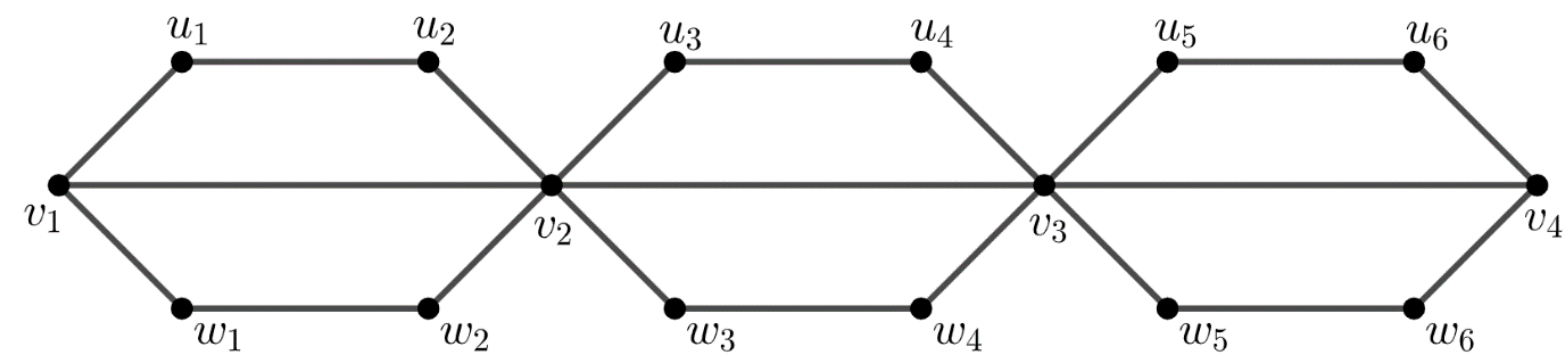

Fig 3. $D Q_{4}$

Definition $2.5^{(14)}$. A double alternative quadrilateral snake $D A Q_{n}$ is obtained from two alternative quadrilateral snakes that have a common path. For example, see Figure 4. 


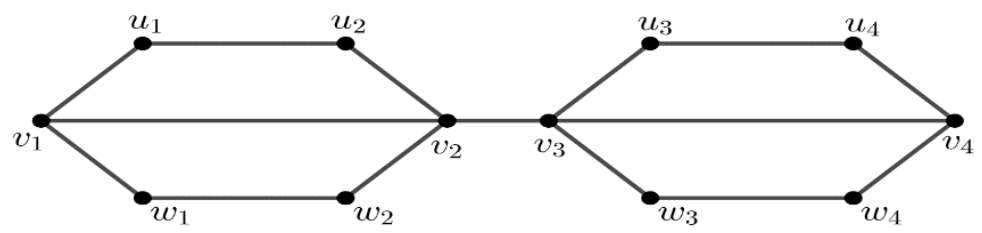

Fig 4. $D A Q_{4}$

\section{Main Results}

The bounds of radio antipodal mean number of quadrilateral snake families are obtained in this section.

Theorem 3.1. The radio antipodal mean number of quadrilateral snakes, $\operatorname{ramn}\left(Q S_{n}\right) \geq 3 n-3, n \geq 4$.

Proof. The graph $Q S_{n}$ has $3 n-2$ vertices. The vertex set of $Q S_{n}$ can be partitioned into two disjoint vertex sets $V_{1}$ and $V_{2}$ such that $V_{1}$ has $2 n-2$ vertices and $V_{2}$ has $n$ vertices. In $V_{1}$ there exists only one pair of vertices at diametric distance. Hence, these vertices are assigned the same label. For the remaining $2 n-4$ vertices, we need at least $2 n-4$ distinct labels. Together with the vertices at diametric distance, we need at least $2 n-3$ labels to label all the vertices in the vertex set $V_{1}$. In $V_{2}$ there exists no pair of vertices at diametric distance and hence we need at least $n$ distinct labels to label all the vertices in $V_{2}$. Therefore, we need at least $3 n-3$ labels to label all the vertices of $Q S_{n}$.

Hence, $\operatorname{ramn}\left(Q S_{n}\right) \geq 3 n-3$.

Theorem 3.2. The radio antipodal mean number of quadrilateral snakes, $\operatorname{ramn}\left(Q S_{n}\right) \leq 4 n-6, n \geq 4$.

Proof. Let $V\left(Q S_{n}\right)$ be the vertex set of $Q S_{n}$. This vertex set can be written as $V\left(Q S_{n}\right)=V_{1} \cup V_{2}$, where $V_{1}=$ $\left\{u_{i}: 1 \leq i \leq 2 n-2\right\}$ and $V_{2}=\left\{v_{i}: 1 \leq i \leq n\right\}$.

In the vertex set $V_{1}$, the vertices $u_{1}$ and $u_{2 n-2}$ are at diametric distance. Therefore, the vertices $u_{1}$ and $u_{2 n-2}$ can receive the same labeling. That is $f\left(u_{1}\right)=f\left(u_{2 n-2}\right)$.

The remaining vertices of $Q S_{n}$ are labelled by the following mapping:

$$
\begin{gathered}
f\left(u_{i}\right)=n+i-2,1 \leq i<2 n-2 \\
f\left(v_{i}\right)=\left\{\begin{aligned}
3 n+i-5,1 \leq i \leq n-2 \\
n-2, i=n-1 \\
4 n-6, \quad i=n
\end{aligned}\right.
\end{gathered}
$$

Claim: The mapping (1) is a valid radio antipodal mean labeling.

Let $u, v \in V\left(Q S_{n}\right)$.

Case 1: Let $u, v \in V_{1}$.

In this case, $d(u, v) \geq 1$.

Case 1.1: Let $u=u_{i}$ and $v=u_{j} 1 \leq i, j<2 n-2, i \neq j$.

Then by (1), $f\left(u_{i}\right)=n+i-2$ and $f\left(u_{j}\right)=n+j-2$.

Therefore, $d(u, v)+\left\lceil\frac{f(u)+f(v)}{2}\right\rceil \geq 1+\left\lceil\frac{2 n+i+j-4}{2}\right\rceil>d$.

Case 1.2: If $u=u_{1}$ and $v=u_{2 n-2}$.

In this case, $d(u, v)=d$. Also $f(u)=n-1$ and $f(v)=n-1$.

Therefore, $d(u, v)+\left\lceil\frac{f(u)+f(v)}{2}\right\rceil \geq d+\left\lceil\frac{2 n-2}{2}\right\rceil \geq d+n-1>d$.

Case 1.3: Let $u=u_{i}$ and $v=u_{2 n-2}, 2 \leq i<2 n-2$.

Then $f\left(u_{i}\right)=n+i-2$ and $f\left(u_{2 n-2}\right)=n-1$.

Therefore, $d(u, v)+\left\lceil\frac{f(u)+f(v)}{2}\right\rceil \geq 1+\left\lceil\frac{2 n+i-3}{2}\right\rceil>d$.

Case 2: If $u, v \in V_{2}$.

In this case, $d(u, v) \geq 1$.

Case 2.1: Let $u=v_{i}$ and $v=v_{j} 1 \leq i, j \leq n-2, i \neq j$.

In this case $d(u, v) \geq 1$.

By mapping (1), $f(u)=3 n+i-5$ and $f(v)=3 n+j-5$.

Therefore, $d(u, v)+\left\lceil\frac{f(u)+f(v)}{2}\right\rceil \geq 1+\left\lceil\frac{6 n+i+j-10}{2}\right\rceil>d$. 
Case 2.2: Let $u=v_{i}, 1 \leq i \leq n-2$ and $v=v_{n-1}$.

Here, $d(u, v) \geq 1$ and by (1), $f(u)=3 n+i-5$ and $f(v)=n-2$.

Therefore, $d(u, v)+\left\lceil\frac{f(u)+f(v)}{2}\right\rceil \geq 1+\left\lceil\frac{4 n+i-7}{2}\right\rceil \geq d$.

Case 2.3: Let $u=v_{i} 1 \leq i \leq n-2$ and $v=v_{n}$.

Here, $d(u, v) \geq 2$ Also, $f(u)=3 n+i-5$ and $f(v)=4 n-6$.

Therefore, $d(u, v)+\left\lceil\frac{f(u)+f(v)}{2}\right\rceil \geq 2+\left\lceil\frac{7 n+i-11}{2}\right\rceil>d$.

Case 2.4: Let $u=v_{n-1}$ and $v=v_{n}$.

Here, $f(u)=n-2$ and $f(v)=4 n-6$. Also, $d(u, v)=1$.

Hence,

$$
d(u, v)+\left\lceil\frac{f(u)+f(v)}{2}\right\rceil \geq 1+\left\lceil\frac{5 n-8}{2}\right\rceil>d
$$

Case 3: Let $u \in V_{1}$ and $v \in V_{2}$.

Case 3.1: Let $u=u_{i}$ and $v=v_{j}, 1 \leq j \leq n-2,1 \leq i<2 n-2$.

Here, $f(u)=n+i-2$ and $f(v)=3 n+j-5$. Also $d(u, v) \geq 1$.

Therefore,

$$
d(u, v)+\left\lceil\frac{f(u)+f(v)}{2}\right\rceil \geq 1+\left\lceil\frac{4 n+i+j-7}{2}\right\rceil>d .
$$

Case 3.2: Let $u \in u_{2 n-2}$ and $v \in v_{n-1}$.

In this case, $d(u, v)=2$ and $f(u)=n-1$ and $f(v)=n-2$.

Therefore,

$$
d(u, v)+\left\lceil\frac{f(u)+f(v)}{2}\right\rceil \geq 2+\left\lceil\frac{2 n-3}{2}\right\rceil \geq d .
$$

Case 3.3: Let $u=u_{2 n-2}$ and $v=v_{n}$.

Here, $d(u, v)=1$. By mapping $(1), f(u)=n-1$ and $f(v)=4 n-6$.

Therefore,

$$
d(u, v)+\left\lceil\frac{f(u)+f(v)}{2}\right\rceil \geq 1+\left\lceil\frac{5 n-7}{2}\right\rceil \geq d .
$$

Case 3.4: Let $u=u_{i}$ and $v=v_{n-1}, 1 \leq i<2 n-2$.

By (1), $f(u)=n+i-2$ and $f(v)=n-2$. In this case, $d(u, v) \geq 1$.

Therefore,

$$
d(u, v)+\left\lceil\frac{f(u)+f(v)}{2}\right\rceil \geq 1+\left\lceil\frac{2 n+i-4}{2}\right\rceil>d .
$$

Case 3.5: Let $u=u_{i}$ and $v=v_{n}, 1 \leq i<2 n-2$.

In this case, $d(u, v) \geq 2$ and $f(u)=n+i-2$ and $f(v)=4 n-6$.

Therefore,

$$
d(u, v)+\left\lceil\frac{f(u)+f(v)}{2}\right\rceil \geq 2+\left\lceil\frac{5 n+i-8}{2}\right\rceil 2 \geq d
$$

Case 3.6: Let $u=u_{2 n-2}$ and $v=v_{i}, 1 \leq i \leq n-2$.

By (1), $f(u)=n-1$ and $f(v)=3 n+i-5$. In this case, $d(u, v) \geq 3$.

Therefore,

$$
d(u, v)+\left\lceil\frac{f(u)+f(v)}{2}\right\rceil \geq 3+\left\lceil\frac{4 n+i-6}{2}\right\rceil>d .
$$

Therefore, the radio antipodal mean labeling condition is satisfied for every pair of vertices in $Q S_{n}$. 
Hence, the mapping (1) is a valid radio antipodal mean labeling.

By the mapping (1), the vertex $v_{n}$ receives the maximum label which is given by $4 n-6$.

Therefore, $\operatorname{ramn}\left(Q S_{n}\right) \leq 4 n-6$.

Theorem 3.3. The bounds of radio antipodal mean number of quadrilateral snakes are given by, $3(n-1) \leq \operatorname{ramn}\left(Q S_{n}\right) \leq$ $2(2 n-3)$.

Proof. The proof follows from Theorem 3.1 and Theorem 3.2.

Theorem 3.4. The radio antipodal mean number of double quadrilateral snakes, $\operatorname{ramn}\left(D Q S_{n}\right) \geq 5 n-6, n \geq 4$.

Proof. The double quadrilateral snake $D Q S_{n}$ has $5 n-4$ vertices. These vertices can be partitioned into three disjoint vertex sets $V_{1}, V_{2}$ and $V_{3}$ where the vertex set $V_{1}$ and $V_{3}$ has $2 n-2$ vertices each and $V_{2}$ has $n$ vertices. Each of the vertex sets $V_{1}$ and $V_{3}$, has one pair of vertices at diametric distance. Hence, these 4 vertices can be labeled with just 2 labels. To label the remaining vertices in $V_{1}$ and $V_{3}$ at least $4 n-8$ labels are needed. Together with the vertices at diametric distance, at least $4 n-6$ labels are needed to label the vertex sets $V_{1}$ and $V_{3}$.

In the vertex set $V_{2}$ there are $n$ vertices and there is no pair of vertices at diametric distance. Therefore, to label the vertex set $V_{2}$, at least $n$ distinct labels are needed.

Hence, the number of labels needed to label all the vertices of $D Q S_{n}$ will be at least $5 n-6$.

Therefore, $\operatorname{ramn}\left(D Q S_{n}\right) \geq 5 n-6, n \geq 4$.

Theorem 3.5. The radio antipodal mean number of double quadrilateral snakes, $\operatorname{ramn}\left(\operatorname{DQS} S_{n} \leq 6 n-9, n \geq 4\right.$.

Proof. Let $V\left(D Q S_{n}\right)$ be the vertex set of $D Q S_{n}$. This vertex set can be partitioned as, $V\left(D Q S_{n}\right)=V_{1} \cup V_{2} \cup V_{3}$, where $V_{1}=\left\{u_{i}: 1 \leq i \leq 2 n-2\right\}, V_{2}=\left\{v_{i}: 1 \leq i \leq n\right\}, V_{3}=\left\{w_{i}: 1 \leq i \leq 2 n-2\right\}$.

In the vertex set $V_{1}$, the vertices $u_{1}$ and $u_{2 n-2}$ are at diametric distance.

Therefore, $f\left(u_{1}\right)=f\left(u_{2 n-2}\right)$.

Similarly, in the vertex set $V_{3}$, the vertex $w_{1}$ and $w_{2 n-2}$ are at diametric distance and hence $f\left(w_{1}\right)=f\left(w_{2 n-2}\right)$.

The remaining vertices of $D Q S_{n}$ are labelled by the mapping:

$$
\begin{gathered}
f\left(u_{i}\right)=n+i-2,1 \leq i<2 n-2 . \\
f\left(v_{i}\right)=\left\{\begin{array}{c}
3 n+i-5,1 \leq i \leq n-2 \\
n-2, i=n-1 \\
3 n+i-6, i=n .
\end{array}\right. \\
f\left(w_{i}\right)=4 n+i-6,1 \leq i<2 n-2 .
\end{gathered}
$$

Claim: The mapping (2) is a valid radio antipodal mean labeling.

Let $u, v$ be any two vertices of $D Q S_{n}$.

Case1: Suppose $u, v \in V_{1}$.

Case 1.1: Let $u=u_{i}$ and $v=u_{j}, i \neq j, 1 \leq i, j<2 n-2$.

In this case, $d\left(u_{i}, u_{j}\right) \geq 1$ and by mapping (2), $f\left(u_{i}\right)=n+i-2$ and $f\left(u_{j}\right)=n+j-2$.

Therefore, $d(u, v)+\left\lceil\frac{f\left(u_{i}\right)+f\left(u_{j}\right)}{2}\right\rceil \geq 1+\left\lceil\frac{2 n+i+j-4}{2}\right\rceil \geq d$.

Case1.2: Let $u=u_{1}$ and $v=u_{2 n-2}$.

Here, $f\left(u_{1}\right)=n-1$ and $f\left(u_{2 n-2}\right)=n-1$.

The distance between the vertices $u_{1}$ and $u_{2 n-2}$ will be $n+1$.

Therefore, $d(u, v)+\left\lceil\frac{f\left(u_{1}\right)+f\left(u_{2 n-2}\right)}{2}\right\rceil \geq(n+1)+\left\lceil\frac{2(n-1)}{2}\right\rceil \geq 2 n>d$.

Case1.3: Let $u=u_{i}$ and $v=u_{2 n-2}, 1 \leq i<2 n-2$.

In this case $d(u, v) \geq 1$. By mapping (2), $f\left(u_{i}\right)=n+i-2$ and $f\left(u_{2 n-2}\right)=n-1$.

Therefore, $d(u, v)+\left\lceil\frac{f\left(u_{i}\right)+f\left(u_{2 n-2}\right)}{2}\right\rceil \geq 1+\left\lceil\frac{2 n+i-3}{2}\right\rceil \geq d$.

Case 2: Suppose $u, v \in V_{2}$.

Case 2.1: Let $u=v_{i}$ and $v=v_{j} 1 \leq i \leq n-2, i \neq j$.

The distance between the vertices $v_{i}$ and $v_{j}$ will be at least 1 .

By mapping (2), $f\left(v_{i}\right)=3 n+i-5$ and $f\left(v_{j}\right)=3 n+j-5$.

Therefore, $d(u, v)+\left\lceil\frac{f(u)+f(v)}{2}\right\rceil \geq 1+\left\lceil\frac{6 n+i+j-10}{2}\right\rceil>d$.

Case 2.2: Let $u=v_{i}, 1 \leq i \leq n-2$ and $v=v_{n-1}$.

In this case, $d(u, v) \geq 1$. Here $f(u)=3 n+i-5$ and $f(v)=n-2$.

Therefore, $d(u, v)+\left\lceil\frac{f(u)+f(v)}{2}\right\rceil \geq 1+\left\lceil\frac{4 n+i-7}{2}\right\rceil \geq d$.

Case 2.3: Let $u=v_{n-1}$ and $v=v_{n}$. 
Here, $d(u, v)=1$. By mapping $(2), f(u)=n-2$ and $f(v)=3 n+i-6$.

Therefore, $d(u, v)+\left\lceil\frac{f(u)+f(v)}{2}\right\rceil \geq 1+\left\lceil\frac{4 n+i-8}{2}\right\rceil \geq d$.

Case 2.4: Let $u=v_{i} \quad 1 \leq i \leq n-2, v=v_{n}$.

The distance between the vertices $u$ and $v$ will be at least 2 .

Here, $f(u)=3 n+i-5$ and $f(v)=3 n+j-6$.

Therefore, $d(u, v)+\left\lceil\frac{f(u)+f(v)}{2}\right\rceil \geq 2+\left\lceil\frac{6 n+i+j-11}{2}\right\rceil \geq d$.

Case 3: Suppose $u, v \in V_{3}$.

This case will be similar to case 1 .

Case 4: Suppose $u \in V_{1}$ and $v \in V_{2}$.

Case 4.1: Let $u=u_{i}, 1 \leq i<2 n-2, v=v_{j}, 1 \leq j \leq n-2$.

Here, $d(u, v) \geq 1$. By mapping (2), $f(u)=n+i-2$ and $f(v)=3 n+j-5$.

Therefore, $d(u, v)+\left\lceil\frac{f(u)+f(v)}{2}\right\rceil \geq 1+\left\lceil\frac{4 n+i+j-7}{2}\right\rceil>d$.

Case 4.2: Let $u=u_{2 n-2}$ and $v=v_{n-1}$.

In this case, $f(u)=n-1$ and $f(v)=n-2$.

The distance between the vertices $u$ and $v$ will be 2 .

Hence, $d(u, v)+\left\lceil\frac{f(u)+f(v)}{2}\right\rceil \geq 2+\left\lceil\frac{2 n-3}{2}\right\rceil \geq d$.

Case 4.3: If $u=u_{1}$ and $v=v_{n-1}$.

Here, $d(u, v)=n-1$ and by mapping (2), $f(u)=n-1$ and $f(v)=n-2$.

Therefore, $d(u, v)+\left\lceil\frac{f(u)+f(v)}{2}\right\rceil \geq n-1+\left\lceil\frac{2 n-3}{2}\right\rceil>d$.

Case 4.4: If $u=u_{i}, 1 \leq i \leq 2 n-2$ and $v=v_{n}$.

The distance between the vertices $u$ and $v$ in this case will be at least 1 . That is

$d(u, v) \geq 1$. By (2), $f(u)=n+i-2$ and $f(v)=3 n+j-6$.

Here, $d(u, v)+\left\lceil\frac{f(u)+f(v)}{2}\right\rceil \geq 1+\left\lceil\frac{4 n+i+j-8}{2}\right\rceil>d$.

Case 5: Suppose $u \in V_{1} v \in V_{3}$.

In this case, $d(u, v) \geq 2$ and $f(u)=n+i-2$ and $f(v)=4 n+j-6$.

Here, $d(u, v)+\left\lceil\frac{f(u)+f(v)}{2}\right\rceil \geq 2+\left\lceil\frac{5 n+i+j-8}{2}\right\rceil>d$.

Case 6: Suppose $u \in V_{2}$ and $v \in V_{3}$.

This case will be like case 4 .

Hence, the radio antipodal mean labeling condition is satisfied by every pair of vertices of $D Q S_{n}$.

Therefore, mapping (2) is a valid radio antipodal mean labeling.

By mapping (2) the vertex $w_{2 n-1}$ receives the maximum label, $f\left(w_{2 n-1}\right)=6 n-9$.

Hence, $\operatorname{ramn}\left(D Q S_{n}\right) \leq 6 n-9$.

Theorem 3.6. The bounds of radio antipodal mean number of double quadrilateral snakes are given by, $5 n-6 \leq$ $\operatorname{ramn}\left(D Q S_{n}\right) \leq 3(2 n-3)$.

Proof. The proof can be obtained directly from Theorem 3.4 and Theorem 3.5.

Theorem 3.7. The radio antipodal mean number of alternate quadrilateral snakes, $\operatorname{ramn}\left(\operatorname{AQS}_{n}\right) \geq 2 n-2, n \equiv$ $1(\bmod 2), n \geq 3$.

Proof. The vertex set of $A Q S_{n}, n \equiv 1(\bmod 2), n \geq 3$ can be partitioned into 2 vertex sets $V_{1}$ and $V_{2}$ such that $\left|V_{1}\right|=n-1$ and $\left|V_{2}\right|=n$.

In $A Q S_{n}$ there is only one pair of vertices that are at diametric distance. Hence, these two vertices can receive the same label. For the remaining $2 n-3$ vertices at least $2 n-3$ distinct labels are needed to label.

Therefore, the number of labels required to label all the vertices of $A Q S_{n}$ will be at least $2 n-2$.

Hence, $\operatorname{ramn}\left(A Q S_{n}\right) \geq 2 n-2, n \equiv 1(\bmod 2), n \geq 3$.

Theorem 3.8. The radio antipodal mean number of alternate quadrilateral snakes, $\operatorname{ramn}\left(A Q S_{n}\right) \leq\left\lceil\frac{n}{2}\right\rceil+2(n-2), n \equiv$ $1(\bmod 2), n \geq 3$.

Proof. Let $V\left(A Q S_{n}\right)$ be the vertex set of $A Q S_{n}$ and can be written as $V\left(A Q S_{n}\right)=V_{1} \cup V_{2}$, where, $V_{1}=\left\{u_{i}: 1 \leq i \leq n-1\right\}$ and $V_{2}=\left\{v_{i}: 1 \leq i \leq n\right\}$.

In the vertex set $V_{1}$, the vertex $u_{1}$ is at diametric distance with the vertices $u_{n-1}$ and $v_{n}$. Without loss of generality, the vertices $u_{1}$ and $v_{n}$ are given the same labeling. That is $f\left(u_{1}\right)=f\left(v_{n}\right)$. 
The remaining vertices of $A Q S_{n}$ are labelled by the following mapping:

$$
\begin{aligned}
& f\left(u_{2 i-1}\right)=n-i-1,1 \leq i \leq\left\lfloor\frac{n}{2}\right\rfloor \\
& f\left(u_{2 i}\right)=n+i-2,1 \leq i \leq\left\lfloor\frac{n}{2}\right\rfloor \\
& f\left(v_{i}\right)=\left\lceil\frac{n}{2}\right\rceil+i+n-3,1 \leq i \leq n-1
\end{aligned}
$$

Claim: The mapping (3) is a valid radio antipodal mean labeling.

Let $u, v$ be any two vertices of $A Q S_{n}$.

The cases are similar to Theorem 3.2.

Hence, the mapping (3) is a valid radio antipodal mean labeling.

By mapping (3), the vertex $v_{n-1}$ receives the maximum label and is given by $\left\lceil\frac{n}{2}\right\rceil+2(n-2)$.

Therefore, $\operatorname{ramn}\left(A Q S_{n}\right) \leq\left\lceil\frac{n}{2}\right\rceil+2(n-2), n \equiv 1(\bmod 2), n \geq 3$.

Theorem 3.9. The bounds of radio antipodal mean number of alternate quadrilateral snakes are given by $2(n-1) \leq$ $\operatorname{ramn}\left(A Q S_{n}\right) \leq\left\lceil\frac{n}{2}\right\rceil+2(n-2), n \equiv 1(\bmod 2)$.

Proof. The proof is obvious from Theorem 3.7 and Theorem 3.8 .

Theorem 3.10. The radio antipodal mean number of alternate quadrilateral snakes, $\operatorname{ramn}\left(A Q S_{n}\right) \geq 2 n-1, n \equiv$ $0(\bmod 2), n \geq 6$.

Proof. The vertex set of $A Q S_{n}$ can be partitioned into 2 vertex sets $V_{1}$ and $V_{2}$ with $n$ vertices each. In $V_{1}$, there is only one pair of vertices that are at diametric distance. Hence, these two vertices can receive the same label. To label the remaining $2 n-2$ vertices, at least $2 n-2$ distinct labels are needed.

Therefore, the number of labels needed to label all the vertices of $A Q S_{n}$ will be at least $2 n-1$.

Hence, $\operatorname{ramn}\left(A Q S_{n}\right) \geq 2 n-1, n \equiv 0(\bmod 2)$.

Theorem 3.11. The radio antipodal mean number of alternate quadrilateral snakes, $\operatorname{ramn}\left(A Q S_{n}\right) \leq 2 n+\left\lfloor\frac{n-1}{2}\right\rfloor, n \equiv$ $0(\bmod 2), n \geq 6$.

Proof. Let $V\left(A Q S_{n}\right)$ be the vertex set of $A Q S_{n}$ and can be written as $V\left(A Q S_{n}\right)=V_{1} \cup V_{2}$, where, $V_{1}=\left\{u_{i}: 1 \leq i \leq n\right\}$ and $V_{2}=\left\{v_{i}: 1 \leq i \leq n\right\}$.

In the vertex set $V_{1}$, the vertices $u_{1}$ and $u_{n}$ are at diametric distance. Therefore, $f\left(u_{1}\right)=f\left(u_{n}\right)$.

The remaining vertices of $A Q S_{n}$ are labeled by the following mapping:

$$
\begin{aligned}
& f\left(u_{2 i-1}\right)=n-(i-1)-1,1 \leq i<\frac{n}{2} \\
& f\left(u_{2 i}\right)=n+i-1,1 \leq i<\frac{n}{2} \\
& f\left(u_{n-1}\right)=n+\left\lfloor\frac{n-1}{2}\right\rfloor \\
& f\left(v_{i}\right)=n+\left\lfloor\frac{n-1}{2}\right\rfloor+i, 1 \leq i \leq n
\end{aligned}
$$

Let $u, v$ be any two vertices of $A Q S_{n}$.

Claim: The mapping (4) is a valid radio antipodal mean labeling.

The cases are similar to Theorem 3.2.

Hence, we conclude that the mapping (4) is a valid radio antipodal mean labeling.

By mapping (4), the vertex $v_{n}$ receives the maximum label and is given by $2 n+\left\lfloor\frac{n-1}{2}\right\rfloor$.

Therefore, $\operatorname{ramn}\left(A Q S_{n}\right) \leq 2 n+\left\lfloor\frac{n-1}{2}\right\rfloor, n \equiv 0(\bmod 2), n \geq 6$.

Theorem 3.12. The bounds of radio antipodal mean number of alternate quadrilateral snakes for $n \equiv 0(\bmod 2)$ are given by, $2 n-1 \leq \operatorname{ramn}\left(A Q S_{n}\right) \leq 2 n+\left\lfloor\frac{n-1}{2}\right\rfloor$.

Proof. The proof follows from Theorem 3.10 and Theorem 3.11.

Theorem 3.13. The radio antipodal mean number of double alternate quadrilateral snakes, $\operatorname{ramn}\left(\operatorname{DAQS}_{n}\right) \geq 3 n-4, n \equiv$ $1(\bmod 2), n \geq 6$.

Proof. The double alternate quadrilateral snake has $3 n-2$ vertices for $n \equiv 1(\bmod 2)$. These vertices can be partitioned into 3 disjoint vertex sets $V_{1}, V_{2}$ and $V_{3}$. The vertex sets $V_{1}$ and $V_{3}$ respectively have $n-1$ vertices and $V_{2}$ has $n$ vertices.

In $D A Q S_{n}$ there are two pairs of vertices are at diametric distance and hence these four vertices can be labeled using two labels. 
To label the remaining $3 n-6$ vertices, at least $3 n-6$ distinct labels are needed. Therefore, the number of labels required to label the vertices of $D A Q S_{n}$ will be at least $3 n-4$.

Hence, $\operatorname{ramn}\left(\operatorname{DAQS}_{n}\right) \geq 3 n-4, n \equiv 1(\bmod 2), n \geq 6$.

Theorem 3.14. The radio antipodal mean number of double alternate quadrilateral snakes, $\operatorname{ramn}\left(\operatorname{DAQS}_{n}\right) \leq 3 n+\left(\frac{n-1}{2}\right)-$ $5, n \equiv 1(\bmod 2), n \geq 3$.

Proof. Let $V\left(D A Q S_{n}\right)$ be the vertex set of $D A Q S_{n}$.

It can be partitioned as, $V\left(D A Q S_{n}\right)=V_{1} \cup V_{2} \cup V_{3}$, where $V_{1}=\left\{u_{i}: 1 \leq i \leq n-1\right\}, V_{2}=\left\{v_{i}: 1 \leq i \leq n\right\}, V_{3}=$ $\left\{w_{i}: 1 \leq i \leq n-1\right\}$.

In $V_{1}$, the vertex $v_{1}$ is at diametric distance from the vertices $u_{n}$ and $v_{n}$.

Without loss of generality, the vertices $u_{1}$ and $v_{n}$ can be given the same label.

Hence, $f\left(u_{1}\right)=f\left(v_{n}\right)$.

Similarly, the vertices $w_{1}$ and $u_{n-1}$ are at diametric distance and hence they can be assigned the same labeling. Therefore, $f\left(w_{1}\right)=f\left(u_{n-1}\right)$.

The remaining vertices of $D A Q S_{n}$ are labeled by the mapping:

$$
\begin{aligned}
& f\left(u_{2 i-1}\right)=n-i-1,1 \leq i \leq\left\lfloor\frac{n}{2}\right\rfloor \\
& f\left(u_{2 i}\right)=n+i-2,1 \leq i \leq\left\lfloor\frac{n}{2}\right\rfloor \\
& f\left(v_{i}\right)=n+\left\lfloor\frac{n}{2}\right\rfloor+i-2,1 \leq i \leq n-1 \\
& f\left(w_{i}\right)=2 n+\frac{n-1}{2}+i-4,1<i \leq n-1
\end{aligned}
$$

Claim: The mapping (5) is valid radio antipodal mean labeling.

Let $u, v$ be any two vertices of $D A Q S_{n}$.

The cases are like Theorem 3.5.

Hence, we conclude that the mapping (5) is valid radio antipodal mean labeling.

By mapping (5), the vertex $w_{n-1}$ receives the maximum label, and is given by $3 n+\left(\frac{n-1}{2}\right)-5$.

Therefore, $\operatorname{ramn}\left(\operatorname{DAQS}_{n}\right) \leq 3 n+\left(\frac{n-1}{2}\right)-5, n \equiv 1(\bmod 2), n \geq 3$.

Theorem 3.15. The bounds of radio antipodal mean number of double alternate quadrilateral snakes for $n \equiv 1(\bmod 2)$ are given by, $3 n-4 \leq \operatorname{ramn}\left(D_{A} Q S_{n}\right) \leq 3 n+\left(\frac{n-1}{2}\right)-5$.

Proof. The proof follows from Theorem 3.13 and Theorem 3.14.

Theorem 3.16. The radio antipodal mean number of double alternate quadrilateral snakes, $\operatorname{ramn}\left(\operatorname{DAQS} S_{n} \geq 3 n-2, n \equiv\right.$ $0(\bmod 2), n \geq 6$.

Proof. The double alternate quadrilateral snake has $3 n$ vertices for $n \equiv 0(\bmod 2)$. These vertices can be partitioned into 3 disjoint vertex sets $V_{1}, V_{2}$ and $V_{3}$ such that each vertex set has $n$ vertices. In $D A Q S_{n}$, two pairs of vertices are at diametric distance and hence these four vertices can be given the same label. To label the remaining $3 n-4$ vertices, at least $3 n-4$ distinct labels are needed.

Therefore, the number of labels needed to label all the vertices of $D A Q S_{n}$ will be at least $3 n-2$.

Hence, $\operatorname{ramn}\left(\operatorname{DAQS}_{n}\right) \geq 3 n-2, n \equiv 0(\bmod 2), n \geq 6$.

Theorem 3.17. The radio antipodal mean number of double alternate quadrilateral snakes, $\operatorname{ramn}\left(\operatorname{DAQS} S_{n} \leq 3 n+\left\lfloor\frac{n-1}{2}\right\rfloor-\right.$ $1, n \equiv 0(\bmod 2), n \geq 6$.

Proof. Let $V\left(D A Q S_{n}\right)$ be the vertex set of $D A Q S_{n}$.

This vertex set can be written as, $V\left(D A Q S_{n}\right)=V_{1} \cup V_{2} \cup V_{3}$, where $V_{1}=\left\{u_{i}: 1 \leq i \leq n\right\}, V_{2}=\left\{v_{i}: 1 \leq i \leq n\right\}, V_{3}=$ $\left\{w_{i}: 1 \leq i \leq n\right\}$.

In $V_{1}$, the vertices $u_{1}$ and $u_{n}$ are at diametric distance. This implies, $f\left(u_{1}\right)=f\left(u_{n}\right)$.

Similarly, in $V_{3}$, the vertices $w_{1}$ and $w_{n}$ are at diametric distance. Therefore, $f\left(w_{1}\right)=f\left(w_{n}\right)$.

The remaining vertices of $D A Q S_{n}$ are labelled by the following mapping:

$$
\begin{aligned}
& f\left(u_{2 i-1}\right)=n-(i-1)-1,1 \leq i<n / 2 \\
& f\left(u_{2 i}\right)=n+i-1,1 \leq i<n / 2 \\
& f\left(u_{n-1}\right)=n+\lfloor(n-1) / 2\rfloor \\
& f\left(v_{i}\right)=n+\lfloor(n-1) / 2\rfloor+i, 1 \leq i \leq n \\
& f\left(w_{i}\right)=2 n+\lfloor(n-1) / 2\rfloor+i, 1 \leq i<n
\end{aligned}
$$


Claim: The mapping (6) is valid radio antipodal mean labeling.

Let $u, v$ be any two vertices of $D A Q S_{n}$.

The cases are similar to Theorem 3.5.

Hence, we conclude that the mapping (6) is valid radio antipodal mean labeling.

By mapping (6), the vertex $W_{n}$ receives the maximum label and is given by $2 n+\left\lfloor\frac{n-1}{2}\right\rfloor$.

Therefore, $\operatorname{ramn}\left(\operatorname{DAQS}_{n}\right) \leq 3 n+\left\lfloor\frac{n-1}{2}\right\rfloor-1, n \equiv 0(\bmod 2), n \geq 6$.

Theorem 3.18. The bounds of radio antipodal mean number of double alternate quadrilateral snakes for $n \equiv 0(\bmod 2)$ are given by, $3 n-2 \leq \operatorname{ramn}\left(D A Q S_{n}\right) \leq 3 n+\left\lfloor\frac{n-1}{2}\right\rfloor-1$.

Proof. The proof is obvious from Theorem 3.16 and Theorem 3.17.

\section{Conclusion}

The channel assignment is important in communication engineering which can be formulated as an optimization problem mathematically. It is an interesting and challenging as all the radio transmitters must be labeled without any interference using minimum span. In this study, the bounds of the radio antipodal mean number of quadrilateral snake families have been investigated. This work can be extended further to other communication networks.

\section{Acknowledgment}

The authors wish to thank the Management of Sri Sivasubramaniya Nadar College of Engineering (Autonomous) for their continuous support and encouragement to do research.

\section{References}

1) Koam ANA, Ahmad A, Haider A. Radio Number Associated with Zero Divisor Graph. Mathematics. 2020;8(12):1-10. Available from: https: //dx.doi.org/10.3390/math8122187.

2) Vaidya SK, Vihol PL. Radio Labeling For Some Cycle Related Graphs. International Journal of Mathematics and Soft Computing. 2012;2(2):11-24. Available from: https://dx.doi.org/10.26708/ijmsc.2012.2.2.03.

3) Arputha TJ, Venugopal P, Giridaran M. RADIO ANTIPODAL MEAN LABELING OF TRIANGULAR SNAKE FAMILIES. Advances in Mathematics: Scientific Journal. 2020;9(11):9739-9746. Available from: https://dx.doi.org/10.37418/amsj.9.11.82.

4) Kang SM, Nazeer S, Nazeer W, Kousar I, Jung CY. Radio Labeling and Radio Number of Caterpillar Related Graphs. Mitteilungen Klosterneuburg. 2015;65(5):149-159.

5) Hale WK. Frequency assignment: theory and applications. Proceedings of the IEEE. 1980;68(12):1497-1514. doi:10.1109/PROC.1980.11899.

6) Havet F. Channel assignment and multicolouring of the induced subgraphs of the triangular lattice. Discrete Mathematics. 2001;233(1-3):219-231. Available from: https://dx.doi.org/10.1016/s0012-365x(00)00241-7.

7) Griggs JR, Yeh RK. Labelling Graphs with a Condition at Distance 2. SIAM Journal on Discrete Mathematics. 1992;5(4):586-595. Available from: https://dx.doi.org/10.1137/0405048.

8) Chartrand G, Erwin D, Zhang P, Harary F. Radio labelings of graphs. Bulletin of the Institute of Combinatorics and its Applications. 2001;33:77-85.

9) Kchikech M, Khennoufa R, Togni O. Linear and cyclic radio k-labelings of trees. Discussiones Mathematicae Graph Theory. 2007;27(1):105-105. Available from: https://dx.doi.org/10.7151/dmgt.1348.

10) Chartrand G, Erwin D, Zhang P. Radio antipodal colorings of graphs. Mathematica Bohemica. 2002;127(1):57-69. Available from: https://dx.doi.org/10. 21136/mb.2002.133978.

11) Ponraj R, Narayanan SS, Kala R. Radio mean labeling of a graph. AKCE International Journal of Graphs and Combinatorics. 2015;12(2-3):224-228. Available from: https://dx.doi.org/10.1016/j.akcej.2015.11.019.

12) Xavier DA, Thivyarathi RC. Radio antipodal mean number of certain graphs. International Journal of Mathematics Trends and Technology. 2018;54(6):467470. doi:10.14445/22315373/IJMTT-V54P556.

13) Yenoke K, T AJ, P V. On The Radio Antipodal Mean Number of Certain Types of Ladder Graphs. International Journal of Innovative Research in Science, Engineering and Technology. 2020;09(06):4607-4614. Available from: https://dx.doi.org/10.15680/ijirset.2020.0906001.

14) Smitha KMB, Thirusangu K. Distance Two Labeling of Quadrilateral Snake Families. International Journal of Pure and Applied Mathematical Sciences. 2016;9(2):283-298. Available from: https://www.ripublication.com/ijpams16/ijpamsv9n2_19.pdf. 